\title{
E864: Experimental Results on Exotic Nuclei and Rare Probes
}

\author{
J.L. Nagle ${ }^{a}$ for the E864 Collaboration \\ aDepartment of Physics, Columbia University \\ New York, NY 10027, USA \\ E-mail: nagle@nevis1.nevis.columbia.edu
}

Experiment 864 at the BNL-AGS was designed to search for exotic states of strange quark matter and other rare composite particles. The experiment was commissioned in 1994 and completed its final run in 1998. Here, we present an overview of the experimental results for the production of light nuclei up to $A=7$, antiprotons and antideuterons, hypernuclei and production limits on new states of quark matter.

\section{INTRODUCTION}

Shortly after the Big Bang the universe had expanded and cooled such that temperatures were low enough for light nuclei (deuterons, ${ }^{3} \mathrm{He}$, tritons, etc.) to coalesce. The present abundance of these states yields significant information confirming our picture of the evolution of the universe from its earliest stages. In a similar manner, we can gain important information about relativistic heavy ion collisions through the study of "little bang" nucleosynthesis.

For over a decade, the source dimensions and flow profile of the dense nuclear matter formed in heavy ion collisions has been studied through the measurement of particle correlations [1]. The multiplicities of light nuclei have similar information since they result from correlations between nucleons when the hadronic system freezes out. In addition, in heavy ion collisions, there is a substantial abundance of antibaryons and strange baryons which may produce more exotic antinuclei and hypernuclei. We present here preliminary results on the yields of these states and production upper limits on meta-stable strange quark matter states.

\section{EXPERIMENT}

Experiment 864 is a two dipole, open-geometry, magnetic spectrometer. We have beam counters to indicate "good" beam on target and give a start time for the interaction. Particles within our acceptance pass through two large aperture dipole magnets and are tracked through a series of time-of-flight hodoscopes and straw tube stations. From the track trajectory we determine the particle momentum, and together with the time information from the hodoscopes, we calculate the particle mass. For the high sensitivity searches even small cross section scattering processes will occur and produce incorrect mass information. A hadronic calorimeter at the end of the spectrometer provides an independent measure of the particle mass through the particle's energy and time-of-flight. 
This detector is also read-out and used for our "late-energy" trigger which effectively selects events in which a high mass candidate is present. Since antibaryons leave additional annihilation energy in the calorimeter, the trigger is also used to enhance the antiproton and antideuteron sample. The experiment in total recorded of order one billion events, which effectively sampled on the order of 50 billion $10 \%$ most central $\mathrm{Au}-\mathrm{Pb} / \mathrm{Pt}$ collisions.

\section{LIGHT NUCLEI}

We have measured the transverse momentum spectra for nucleons (protons and neutrons) and light nuclei up to baryon number $\mathrm{A}=3$ at $y=2.3$. The spectra show an increasing Boltzmann temperature parameter with increasing mass (proton $\mathrm{T}=212 \pm 16 \mathrm{MeV}$, neutron $T=223 \pm 23 \mathrm{MeV}$, deuteron $\mathrm{T}=347 \pm 17 \mathrm{MeV},{ }^{3} \mathrm{He} T=405 \pm 24 \mathrm{MeV}$ ). This feature would be unexpected from an isotropic fireball in thermal equilibrium; however, it has previously been observed that transverse expansion or flow results in increased "temperature" parameters for heavier states. Polleri et al. have shown [2] that the heavier nuclear states give significantly more information on the density profile and flow velocity than can be derived from lighter states $(\pi, K, p)$. Detailed comparisons between our data and such models should constrain our picture of the flow evolution.

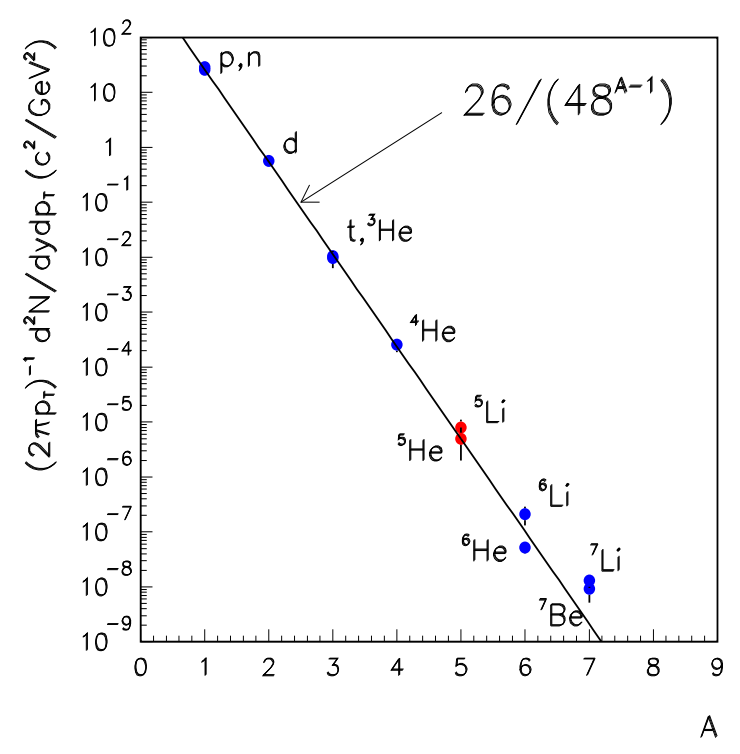

Figure 1. Invariant yields per central collision at or near $y=1.9, p_{T} / A=$ $200 \mathrm{MeV} / \mathrm{c}$ as a function of mass number $A$.

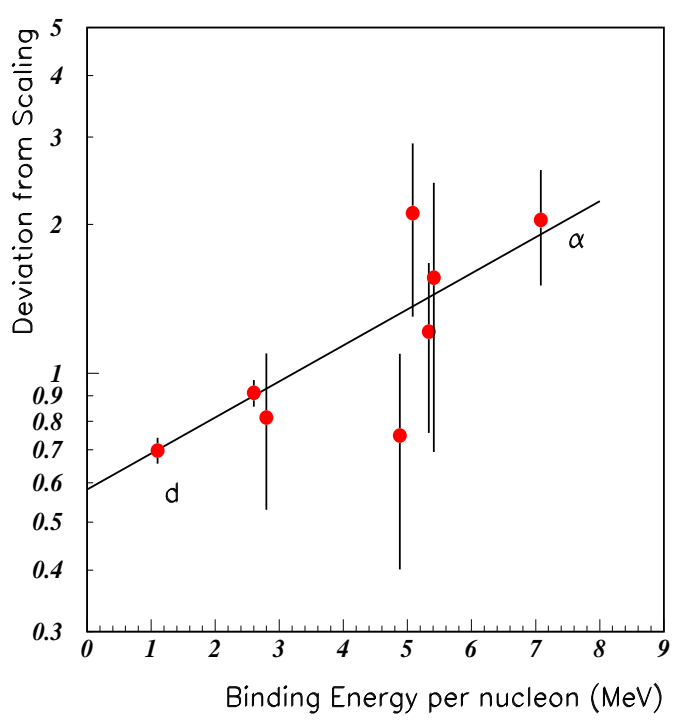

Figure 2. Relative deviations from scaling relation after correction for baryon number $A$, isospin $I_{z}$ and spin $J$ as a function of binding energy per nucleon $(\mathrm{MeV})$.

Using the high-mass trigger, we are also able to measure the invariant yields of light nuclei which are produced down to the level of a few per billion central interactions. We show in Figure 11 the measured yields of light nuclei up to baryon number $A=7$ as a 
function of baryon number. We observe a striking exponential behavior where the yields are characterized by a penalty factor of approximately 48 per additional nucleon in the cluster. It is interesting to note that in the early universe the lack of a stable $A=5$ state has a large impact on the higher mass cluster yields. However, since ${ }^{5} \mathrm{Li}$ and ${ }^{5} \mathrm{He}$ have lifetimes longer than the entire time evolution of the heavy ion collision, their yields appear to roughly scale with the other stable nuclei. We are able to measure these states through a two particle reconstruction of the ${ }^{4} \mathrm{He}$ and $p$ or $n$ and appropriate background subtraction.

Although the data are reasonably described by this simple scaling over ten order of magnitude in yield, there are deviations. There are some easily understood deviations from scaling due to differences in isospin and spin factors. In addition, as shown in Figure 2, it appears that there is a dependence on the binding energy per nucleon of the nuclear state. The weakly bound deuteron appears to have a suppressed yield relative to the most bound state that we measure, the alpha particle. This dependence on how tightly bound the object is may be an autocorrelation with the dependence on the nuclear size.

\section{ANTINUCLEI}

The production of antibaryons has long been recognized as a powerful tool for the study of the baryon dense heavy ion collision environment. At AGS energies antiproton production is suppressed in initial collisions and is further reduced due to their high annihilation cross section. Antideuterons are actually below threshold in nucleon-nucleon collisions at the $11.6 \mathrm{~A} \mathrm{GeV} / \mathrm{c}$ applicable for our experiment. Thus, it is expected that antideuterons are formed from the coalescence of antiprotons and antineutrons at the freeze out surface.

It has been theorized that due to the large absorption, antibaryons are emitted only on the surface of the collision volume. This unusual emission surface results in a suppressed yield of antinuclei [3]. We present here the first measurement of antideuterons in $A u$ induced collisions at the AGS. We show in Figure 3 the coalescence scale factor $\overline{B_{2}}$ (the ratio of antideuterons to antiprotons squared) which appears to be almost an order of magnitude below the rate for deuterons $B_{2}$; thus leading one to conclude that surface emission may be a correct hypothesis.

However, we have previously published a surprising result on the yield of strange antibaryons relative to antiprotons [4]. E864 measures antiprotons which include those that result from the decay of strange antibaryons $(\bar{\Lambda}, \bar{\Sigma}, \Xi$, etc.). When we compare our results to those of experiment E878 which measures only primordial antiprotons, we observe a statistically significant difference. This difference can be attributed to the decay products of strange antibaryons. Thus, we have calculated the ratio of strange antibaryons to antiprotons near midrapidity and low transverse momentum (in the region of the experimental acceptance) as a function of centrality, shown in Figure 4 . The implied ratio of $\bar{Y} / \bar{p}=3.5$ for the most central collisions is much above the ratio from $p-p$ and $e^{+}-e^{-}$ collisions of $\bar{Y} / \bar{p}=0.2-0.4$. This large ratio appears to be confirmed by preliminary E917 results shown at this conference [5].

If we consider that, in fact, most of the antiprotons included in the denominator of the 


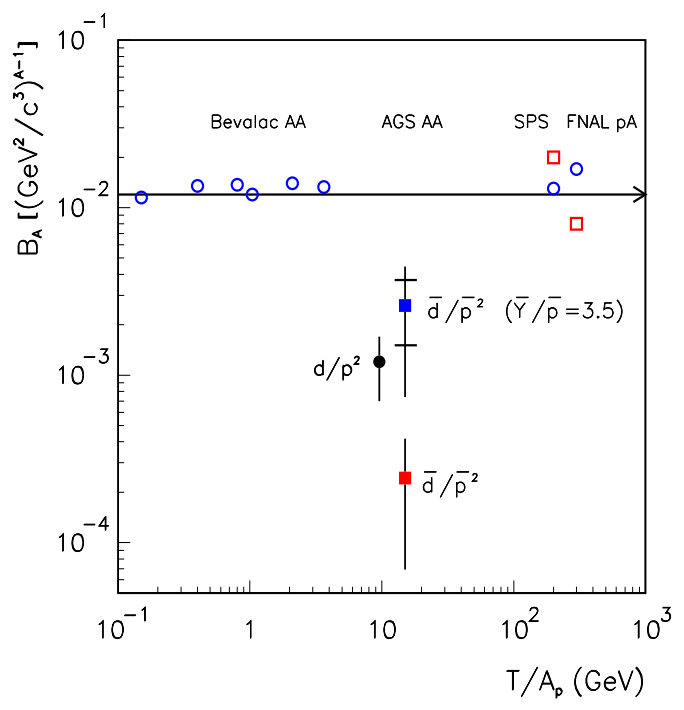

Figure 3. Preliminary coalescence scale factor $\overline{B_{2}}$ for antideuterons using our measured antiproton yield and also using the antiprotons removing the contribution from strange antibaryons. The corrected value agrees with our measured value for $B_{2}$ for deuterons.

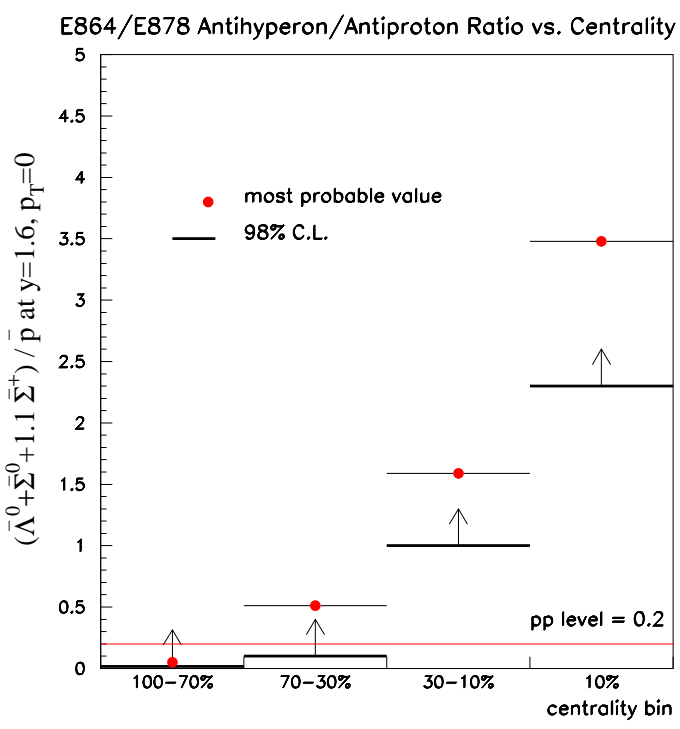

Figure 4. The inferred ratio of strange antibaryons to antiprotons at midrapidity and low transverse momentum as a function of collision centrality. These results are calculated from the antiproton measurements from E878 and E864.

antideuteron scale factor are really strange antibaryons, then we have underestimated the $\overline{B_{2}}$ value. Since strange antibaryons decay long after the coalescence process has taken place, they do not contribute to the yield of antideuterons. If we correct the $\overline{B_{2}}$ value for these contributions, then it increases the scale factor by over an order of magnitude and into agreement within statistical and systematic errors of the deuteron scale factor. Both values are below the $B_{2}$ values from low energy $A-A$ collisions and high energy $p-p$ collisions, indicating a much larger freeze out volume for this system.

\section{HYPERNUCLEI}

Unlike the early universe, the heavy ion system freezes out on a time scale $10-20 \mathrm{fm} / \mathrm{c}$, on the order of the strong interaction. Therefore, there are a substantial number of strange baryons which have not decayed weakly that contribute to a "strange" nucleosynthesis process. Here we have searched for the state ${ }_{\Lambda}^{3} H$ via its decay mode to ${ }^{3} \mathrm{He}+\pi$. We have a preliminary analysis of $2 / 3$ of the relevant data sample and observe a signal at approximately the $2 \sigma$ level. We measure a preliminary invariant yield of ${ }_{\Lambda}^{3} H$ in the kinematic range $1.6<y<2.8$ and $0<p_{T}<2 \mathrm{GeV}$ of $2.6 \pm 1.4 \times 10^{-4} c^{2} / \mathrm{GeV}^{2}$. Dover et al. have estimated that there is an additional penalty factor for adding a strange baryon (instead of non-strange) to a nucleus of $1 / 3$ due to the smaller abundance of strange baryons [9]. Using the above measured invariant yield of ${ }_{\Lambda}^{3} H$ combined with our measurements of p, n, ${ }^{3} \mathrm{He}$, and the E891 measure of $\Lambda$ [10], we can check this model. Our preliminary results show that in addition to the penalty for the smaller abundance 


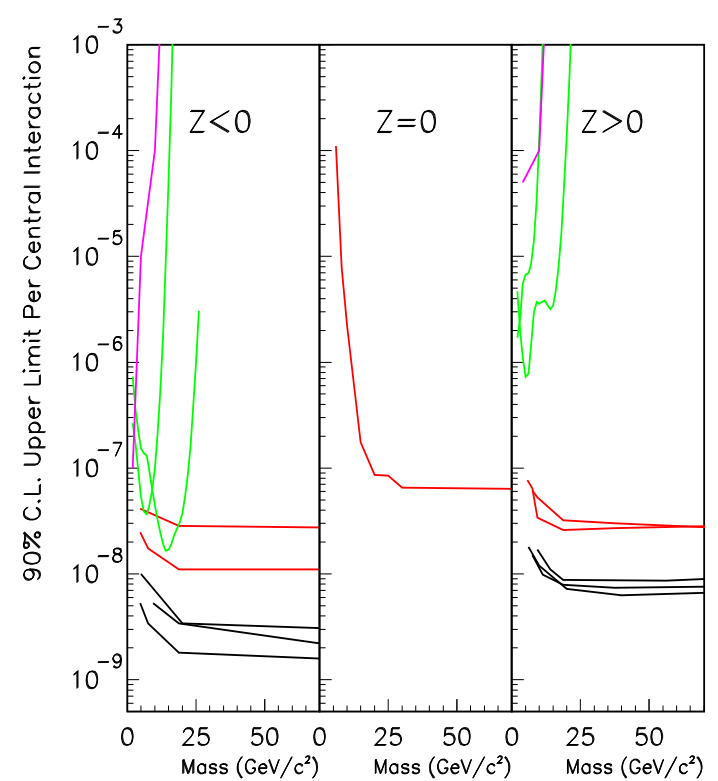

Figure 5. 90\% Confidence Level Upper Limits on the production of strange quark matter (negative, positive and neutral charged states). The upper curves at low mass are from E878 and E886. The lower gray curves are the E864 published results and even lower black curves are the preliminary E864 final results.

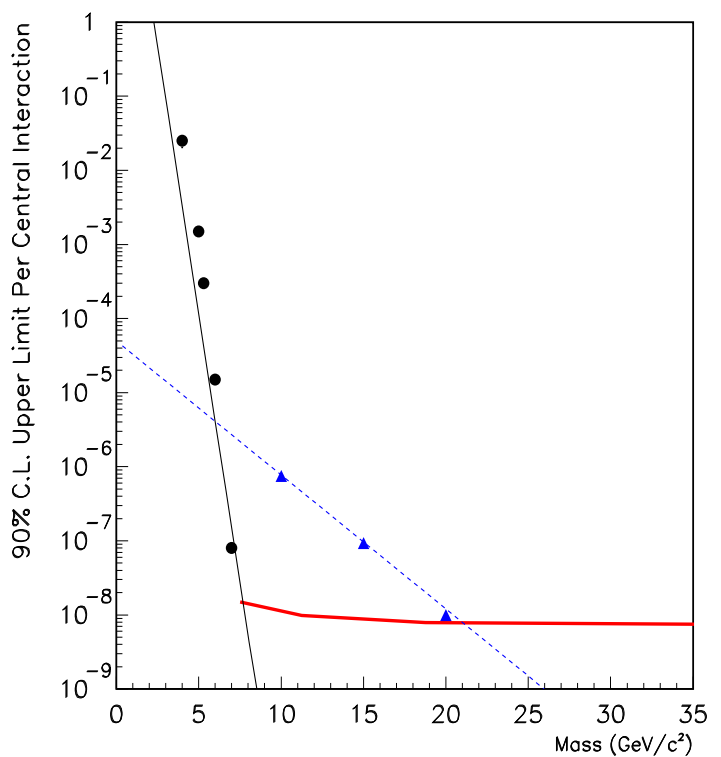

Figure 6. 90\% Confidence Level Upper Limits on the production of $Z=$ +2 SQM. Also shown are predictions for their production in a cooling quark plasma (triangles) and via hypernuclei acting as doorway states (circles).

of $\Lambda$, there is an additional suppression of $0.162 \pm 0.088$. This factor indicates that it is more difficult to make strange clusters than originally predicted. This suppression factor may imply that production of the $H$ di-baryon and other strange quark matter states via coalescence will also be suppressed.

\section{STRANGE QUARK MATTER}

It has been theorized that strange quark matter (SQM), composed of nearly equal numbers of up, down and strange quarks, might be meta-stable or even completely stable in bulk. Heavy ion collisions are the best known environment for the production of small states of strange quark matter. The collisions produce a large number of strange quarks in a relatively small configuration space. In addition, the possible transition to a deconfined quark-gluon plasma is a natural environment to give rise to such new quark composites.

We have not observed any evidence for meta stable (lifetimes of order $50 \mathrm{~ns}$ or greater) charged or neutral $Z= \pm 0,1,2,3,>3$ SQM states. We have previously published $90 \%$ confidence level upper limits based on data taken in 1995 [6] 8]. Here we present our final limits on charged states using our complete data sample. Shown in Figure 5 are the upper limits from Experiments E878 and E886 in addition to our published results (grey lines). Our final limits (black lines) show that we rule out the production of SQM at the level of a $2-9 \times 10^{-9}$ per $10 \%$ central collision. 
With the completion of the fixed target program at the BNL-AGS and the soon to be published final limits from Experiment E864, it is an appropriate time to ask what is the significance in the absence of any observation. Do these results imply that SQM in the mass range $A=3-100$ is not meta stable? In order to answer this question, we must examine the various production models for the creation of strangelets in these reactions.

Our results rule out a number of published predictions [11 for SQM production in a plasma scenario as shown in Figure 6; however, these model calculations are not well constrained. There are also models which predict the rate of SQM production assuming that multi-strange hypernuclei are formed via coalescence at hadronic freeze out. If a SQM state with the same quantum numbers is more stable than this hypernucleus, the hypernucleus may act as a "doorway" and transition to the quark matter state. Using the number from [9], our experiment is sensitive to states of baryon number $A=6-7$ and strangeness $S=2-3$. However, in light of our preliminary measure of hypernuclei production, this would appear to overestimate our sensitivity.

\section{SUMMARY}

E864 has completed the most sensitive search in relativistic heavy ion collisions at the AGS for exotic nuclear states and novel quark composites (strange quark matter) to date. We observe no SQM and thus set the most stringent upper limits on its production. We have extended the study of coalescence to a new regime including nuclei up to $A=$ 7, unstable nuclei ${ }^{5} \mathrm{He},{ }^{5} \mathrm{Li}$, hypernuclei ${ }_{\Lambda}^{3} \mathrm{H}$, and antideuterons. The overall scaling of nuclear yields, the suppression of hypernuclei, and the extreme enhancement of strange antibaryons present a challenge to the theoretical community.

\section{ACKNOWLEDGMENTS}

We gratefully acknowledge the efforts of the AGS staff and support from the DOE High Energy and Nuclear Physics Divisions and the NSF.

\section{REFERENCES}

1. U. Wiedemann and U. Heinz, CU-TP-931, nucl-th/9901094 (1999).

2. A. Polleri, in these proceedings, A. Polleri et. al., Phys. Lett. B., 419 (1998) 19.

3. S. Mrowczynski, Phys. Lett. B., 248 (1990) 459.

4. T.A. Armstrong et al., Phys. Rev. Lett. 79, 3351 (1997). T.A. Armstrong et al., Phys. Rev. C59, 2699 (1999).

5. D. Hofman for the E917 collaboration, in these proceedings.

6. T.A. Armstrong et al., Phys. Rev. Lett. 79, 3612 (1997).

7. T.A. Armstrong et al., Nucl. Phys A625, 494 (1997).

8. T.A. Armstrong et al., Phys. Rev. C59, 1829 (1999).

9. A. J. Baltz et al., Phys. Lett. B, 325 (1994) 7.

10. S. Ahmad et al., Phys. Lett. B, 382 (1996) 35.

11. H. Crawford et al., Phys. Rev. D 45, 857 (1992) 